\title{
How the Most Trusted Venues for Health-Related Information Influence Physician Referrals to Smoking Cessation Services
}

\author{
Judy Kruger, Angela Trosclair, Crystal Bruce, and Diane Beistle \\ Office on Smoking and Health, Centers for Disease Control and Prevention, 4770 Buford Highway NE, \\ K-50, Atlanta, GA 30341-3717, USA \\ Correspondence should be addressed to Judy Kruger, ezk0@cdc.gov
}

Received 29 April 2012; Accepted 21 May 2012

Academic Editors: P. Bendtsen, C. M. Buchalla, S. Gallus, and W. Kirch

Copyright ( 2012 Judy Kruger et al. This is an open access article distributed under the Creative Commons Attribution License, which permits unrestricted use, distribution, and reproduction in any medium, provided the original work is properly cited.

Public health programs seek to educate physicians by using a variety of venues. Therefore, it is important to understand which health information sources physicians are using and how these sources affect referrals. We explored how venues for health-related information affect physicians' referral practices to smoking cessation services. The 2008 DocStyles survey asked physicians to rank a list of their most trusted sources of health-related information. The analysis was restricted to 1,617 physicians who responded to all variables of interest. In this sample, the most trusted sources of health-related information cited by physicians were medical journals (95.9\%), government health agencies $(82.2 \%)$, other physicians $(76.4 \%)$, professional medical societies $(75.2 \%)$, and medical Web sites or podcasts $(65.9 \%)$. Medical providers were more likely to refer tobacco users to cessation services if they used professional medical societies as a source to obtain patient health-related information, compared with medical providers not using this source (multivariate odds ratio $=1.31 ; 95 \%$ confidence interval $=1.03-1.66$ ). Physicians use many health information sources. Therefore, to reach physicians effectively, a broad dissemination of guidelines and best practices in tobacco control is needed and should include information for medical societies.

\section{Introduction}

Tobacco use remains the single most preventable cause of disease and death in the United States. In 2010, 19.3\% of the adult population reported smoking every day or some days [1]. Physicians are one of the most important sources of information about smoking cessation for patients who use tobacco [2]. Approximately $84 \%$ of the US population visit a primary care provider each year, and they average 2.1 visits a year, which provide ample opportunity for physicians and their staff to deliver brief, effective, tobacco cessation interventions to their patients who use tobacco [3]. Physician advice increases the number of patients who attempt to quit smoking and succeed $[4,5]$.

During the office medical visit or at the patient's bedside, physicians are frontline educators and treatment providers, and the U.S. Public Health Service (PHS) guidelines confirm the physician's role as essential for providing tobacco dependence services [3]. The PHS clinical practice guidelines for tobacco use and dependence recommend that physicians follow the 5 A's when helping patients quit: ask, advise, assess, assist, and arrange followup [3]. Unfortunately, physicians do not routinely follow all of these guidelines. A report about physician behavior related to smoking cessation by the Association of American Medical Colleges (AAMC) includes physicians in the specialty fields of family medicine, general internal medicine, obstetrics/gynecology, and psychiatry. The AAMC report states the following: $84 \%$ of these physicians report that they routinely ask patients about smoking status; $86 \%$ advise smokers to stop smoking; $63 \%$ assess patients' willingness to quit; $13 \%$ usually refer smokers to others for appropriate treatment; $17 \%$ usually arrange for follow-up visits to address smoking [2]. In addition, the report found that among all specialty groups, few physicians discussed counseling options, enlisted support for quitting, or monitored patient progress in cessation attempts. Physicians reported barriers to providing help, including inadequate knowledge and skills for delivering smoking cessation interventions because of limited medical education and training [2]. In addition, physicians felt that their smoking cessation activities were inadequate because of insufficient organizational resources to help patients quit, 
including a lack of pamphlets for waiting rooms, and onsite group, or individual counseling programs [2]. Physicians frequently mention this lack of knowledge and training for smoking cessation as a barrier to providing effective tobacco cessation treatments $[3,6]$. Additional barriers to counseling patients that physicians have identified are a lack of patient interest, a lack of time allowed during patient appointments, and insufficient compensation [7].

For the types of resources that physicians are using for information, there has been an increase in electronic sources (e.g., medical outcomes software, proprietary access services, the Internet, and CD-ROM educational materials) [8-10]. Medical journal information is now available in both print and on the Web, and physicians are using the Internet to seek answers to their health-related questions [11]. Technology has the potential to provide health care providers with easy access to health information when needed.

It is important to understand which health information venues physicians are using and how these outlets affect physician referral patterns. This understanding can help public health programs focus on tobacco control, target their information to the most appropriate media outlet, and increase use of effective cessation strategies. How physicians are using health-related information venues may guide communication efforts and identify communication strategies for dissemination of tobacco cessation information. The objective of this paper is to explore the effect of health-related information venues on physicians' smoking cessation referral practices.

\section{Methods}

2.1. DocStyles Survey. We selected our study population from respondents to the 2008 DocStyles survey, which was conducted by Porter Novelli, a social marketing and public relations firm. DocStyles is an annual web survey that provides insight into physicians' attitudes, behaviors, knowledge, and counseling behaviors on health issues, and the survey assessed their use of and trust in available health information sources. The sampling was conducted by Epocrates, Inc., by using respondents identified from the Epocrates Honors Panel, an opt-in, verified panel of 135,000 medical practitioners. The primary recruitment method was based on health care providers' self-selection to join the panel and complete the online health care survey at http://www.epocrates.com/honors, after receiving an initial email from Epocrates.

Eligible physicians were verified by checking each by first name, last name, date of birth, medical school, and graduation date against the American Medical Association's (AMA) master file at the time of panel registration. Physicians were screened to include only those who practice in the United States; actively see patients; work in an individual, group, or hospital practice; have been practicing medicine for at least 3 years. Epocrates randomly selected a sample of eligible physicians from their main database to load into their invitation database. The sample was drawn to match AMA master file proportions for age, gender, and region. Physicians were paid an honorarium of $\$ 50-\$ 75$ for completing the survey. Respondents were not required to participate in the 140 question survey, which had multiple subparts designed to provide insights into physicians' counseling behaviors, and were able to exit the survey at any point. For Epocrates to reach their quotas, 14,346 physicians were invited to participate. The final sample consisted of 1,880 respondents, which resulted in a response rate of $22 \%$ after adjusting for individuals who were accepted but then excluded because the quota was filled.

\subsection{Measures}

2.2.1. Demographics and Source of Health-Related Information. Demographic characteristics used in the analysis included gender, age $(18-35,36-45,46-55$, or $\geq 56$ years $)$, and race/ethnicity (non-Hispanic white, non-Hispanic black, Hispanic, non-Hispanic Asian, other). Smoking status was dichotomized into smokers (1-7 days/week) and nonsmokers (0 days/week). Participants were asked about the frequency with which they referred patients to cessation programs: How often, if ever, do you refer your patients who smoke tobacco or use other tobacco products to cessation programs such as a telephone quitline, a smoking cessation class, or one-on-one counseling? Response options included never, rarely, sometimes, usually, and always, and these responses were dichotomized as always/usually/sometimes or never/rarely.

The 2008 DocStyles survey included a series of questions about respondents' use of health-related information. Respondents were asked a screening question for 16 different health information sources: (1) advertising; (2) books; (3) brochures, flyers, pamphlets, and posters; (4) government health agencies; (5) pharmaceutical companies; (6) medical Web sites or medical podcasts; (7) magazine stories or articles; (8) medical journals; (9) newspaper stories or articles; (10) nurses, nurse practitioners, and physician assistants; (11) other physicians; (12) patient advocacy groups; (13) patients or parents who have researched a condition; (14) professional medical societies; (15) radio; (16) television. The question was as follows: please indicate how often you use each of the following sources to obtain patient health-related information. Response options were never, rarely, sometimes, often, and regularly. If they did not answer never to all sources, then they were asked to select five of their most trusted patient health-related information sources from the list of 16 health information sources.

2.3. Statistical Analyses. Descriptive analyses were conducted to characterize physicians by their gender, age, race/ethnicity, smoking status, and referral of patients who use tobacco to cessation services. We used a chi-square test to examine within-group differences in the proportion of physicians who stated that they always/usually/sometimes referred tobacco users to cessation services, compared with those who never/rarely referred to cessation services. To describe the most trusted sources of medical information among physicians, we stratified respondents by gender, age, and 
race/ethnicity to examine the five most trusted sources of health-related information. Separate logistic regression models were used to test the relationship between use of one of the five most trusted health-related information sources and referral to smoking cessation services, including gender, age, and race/ethnicity as covariates. Odds ratios with 95\% confidence intervals (CIs) were calculated to compare referral to cessation services among those who reported the source as a trusted source versus those who did not select the source as one of their most trusted sources. All of the analyses were completed by using SAS version 9.2 (SAS Institute Inc., Cary, NC) software.

\section{Results}

Of the physicians who responded to the survey and all the variables of interest, $68.9 \%$ were men and $31.1 \%$ were women (Table 1). Approximately 40\% (39.4\%) were aged 36-45 years, and $72.9 \%$ were white. More than 93\% (93.4\%) were nonsmokers, and $42.7 \%$ always, usually, or sometimes referred tobacco users to cessation services.

Table 2 shows the proportion of physicians who referred their tobacco-using patients to cessation services. A significant difference in the distribution for referring versus not referring was found for sex and race/ethnicity $(P<.05)$. Statistically significant differences were also found by age among physicians who always, usually, or sometimes referred tobacco users to cessation services compared with those who never or rarely referred tobacco users $(P<.001)$.

In this sample, the most trusted sources of healthrelated information cited by physicians (in rank order) were as follows: medical journals (95.6\%), government health agencies $(82.7 \%)$, other physicians $(76.4 \%)$, professional medical societies $(75.2 \%)$, and medical Web sites or podcasts $(65.9 \%)$. Table 3 presents frequencies by gender, age, and race/ethnicity among physicians and showed that a higher proportion of female physicians than male physicians reported using government health agency information and medical Web sites or podcasts $(P<.001)$. A higher proportion of male physicians $(78.0 \%)$ compared to female physicians $(69.0 \%)$ reported using professional medical societies as a source of health-related information $(P<.001)$. Hispanic and non-Hispanic Asian physicians used government health agency information less than their non-Hispanic black and non-Hispanic white counterparts $(P<.05)$. A lower proportion of non-Hispanic Asian physicians (69.8\%) compared to non-Hispanic white physicians $(77.3 \%)$ reported using professional medical societies $(P<.05)$. Non-Hispanic Asian physicians used medical Web sites or podcasts more than non-Hispanic white $(63.7 \%)$ and non-Hispanic black (66.7\%) physicians $(P<.05)$.

Logistic regression analyses indicated that physician use of selected health-related information sources had an increased likelihood of referring patients to smoking cessation services (Table 4). In the unadjusted model, physicians who used professional medical societies were more likely to refer smokers to smoking cessation services than those who did not (OR1.29; 95\% CI $=1.02-1.63)$. Physicians who
TABLE 1: Selected characteristics of physicians-DocStyles Survey, 2008.

\begin{tabular}{lccc}
\hline Characteristics & $N$ & $\%$ & $95 \%$ CI \\
\hline Gender & & & \\
$\quad$ Men & 1,114 & 68.9 & $66.6-71.1$ \\
$\quad$ Women & 503 & 31.1 & $28.9-33.4$ \\
Age (y) & & & \\
$\quad$ 18-35 & 327 & 20.2 & $18.3-22.3$ \\
$36-45$ & 637 & 39.4 & $37.0-41.8$ \\
$46-55$ & 452 & 28.0 & $25.8-30.2$ \\
56+ & 201 & 12.4 & $10.9-14.1$ \\
Race/ethnicity & & & \\
$\quad$ Non-Hispanic white & 1,179 & 72.9 & $70.7-75.0$ \\
$\quad$ Non-Hispanic black & 48 & 3.0 & $2.2-3.9$ \\
Hispanic & 69 & 4.3 & $3.4-5.4$ \\
$\quad$ Non-Hispanic asian & 248 & 15.3 & $13.7-17.2$ \\
$\quad$ Non-Hispanic other & 73 & 4.5 & $3.6-5.6$ \\
Smoking status & & & \\
$\quad$ Smoker & 107 & 6.6 & $5.5-7.9$ \\
$\quad$ Nonsmoker & 1,510 & 93.4 & $92.1-94.5$ \\
Refers to smoking cessation & & & \\
services & & & \\
Always/usually/sometimes & 690 & 42.7 & $40.3-45.1$ \\
$\quad$ Never/rarely & 927 & 57.3 & $54.9-59.7$ \\
\hline Total & 1,617 & - & - \\
\hline
\end{tabular}

${ }^{a}$ Smoking status was dichotomized into smokers (1-7 days/week) and nonsmokers (0 days/week).

${ }^{\mathrm{b}}$ Cessation services consist of telephone quitline, a smoking cessation class, or one-on-one counseling.

used medical Web sites or podcasts were more likely to refer tobacco users to cessation services than those who did not $(\mathrm{OR}=1.25 ; 95 \% \mathrm{CI}=1.01-1.54)$. When adjusting for sex, age, and race/ethnicity, physicians were more likely refer tobacco users to cessation services if they used professional medical societies as a source to obtain patient health-related information, compared with medical providers not using this source $(\mathrm{AOR}=1.31 ; 95 \% \mathrm{CI}=1.03-1.66)$.

\section{Discussion and Summary}

It has been recommended as standard medical practice for physicians to engage in the 5 A's model for treating tobacco use and dependence: ask, advise, assess, assist, and arrange $[3,5]$. However, referral to cessation services is only a part of the 5 A's. Prior research has shown that physician advice for cessation increases quitting among their patients $[3,4,12]$. One study found that $42.7 \%$ of physicians referred their tobacco-using patients to cessation services. Because physicians supply highly valued advice and can potentially reach many patients multiple times, information about what influences referral patterns may lead to patient behavior change. Tobacco-using patients especially can maximize the benefits of cessation services because free telephone quitline 
TABle 2: Percentage of physicians who refer their tobacco-using patients to cessation services ${ }^{\mathrm{a}}$-DocStyles Survey, 2008.

\begin{tabular}{|c|c|c|c|}
\hline & \multicolumn{3}{|c|}{ Refers to cessation services ${ }^{a}$} \\
\hline & $N$ & $\%$ & $95 \% \mathrm{CI}$ \\
\hline \multicolumn{4}{|l|}{ Gender $^{\mathrm{c}}$} \\
\hline Male & 474 & 42.5 & $(39.7-45.5)$ \\
\hline Female & 216 & 42.9 & $(38.7-47.3)$ \\
\hline \multicolumn{4}{|l|}{ Age $(y)^{d}$} \\
\hline $18-35$ & 128 & 39.1 & $(34.0-44.5)$ \\
\hline $36-45$ & 291 & 45.7 & $(41.8-49.6)$ \\
\hline $46-55$ & 200 & 44.2 & $(39.7-48.9)$ \\
\hline $56+$ & 71 & 35.3 & $(29.0-42.2)$ \\
\hline \multicolumn{4}{|l|}{ Race/ethnicity ${ }^{\mathrm{c}}$} \\
\hline Non-Hispanic white & 485 & 41.1 & $(38.4-44.0)$ \\
\hline Non-Hispanic black & $20^{\mathrm{b}}$ & 41.7 & $(28.7-55.9)$ \\
\hline Hispanic & $35^{\mathrm{b}}$ & 50.7 & $(39.1-62.3)$ \\
\hline Non-Hispanic asian & 113 & 45.6 & $(39.5-51.8)$ \\
\hline Non-Hispanic other & $37^{\mathrm{b}}$ & 50.7 & $(39.4-61.9)$ \\
\hline Total & 690 & 42.7 & $(40.3-45.1)$ \\
\hline \multicolumn{4}{|c|}{ 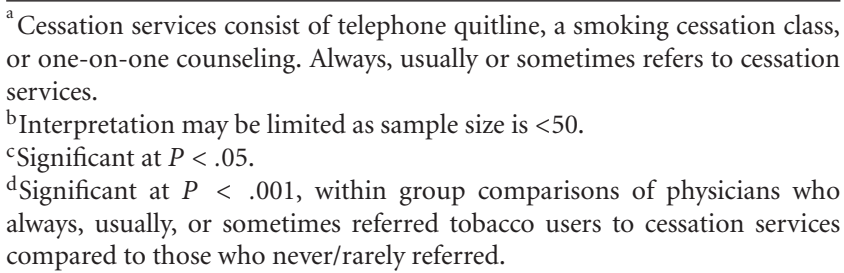 } \\
\hline
\end{tabular}

support services are available to help individuals who want to stop smoking or using tobacco.

Physicians used multiple venues to obtain health-related information, and referrals to cessation services were related to their most trusted sources of health-related information used in this sample. Our study found that physicians who obtained their most trusted health-related information from professional medical societies had an increased likelihood of referring patients to smoking cessation services. Several medical organizations such the American Academy of Pediatrics [13], the American College of Obstetricians and Gynecologist [14], the American Medical Association [15], and the American Academy of Family Physicians [16] recommend routinely assessing tobacco use and referral of patients to cessation services. Because of the influence that professional medical societies and their recommendations have on physicians' referral practices, specialty societies should continue to reach physicians with cessation information through multiple avenues simultaneously. Health communicators wishing to reach physicians with cessation information should consider these professional medical associations when developing their communication plans.

Electronic sources are growing in popularity and are currently used by physicians $53 \%$ of the time to seek answers to their health questions, including cessation advice [17]. In addition, physicians are turning to the Internet to access Continuing Medical Education (CME) online health information [18]. Findings from our study suggest that medical Web sites or podcasts are a trusted source of healthrelated information and may influence referral to smoking cessation services. In a review of physician informationseeking behaviors by Wilson and colleagues, the authors found general medical podcasts to be a valuable resource for providing an overview of the latest research on a particular topic, as well as opportunities to explore areas outside of a physician's core discipline [10]. Further research on Internet tools designed for physicians such as medical Web sites and podcasts can help health communicators develop best practice models and disseminate health-related information more effectively.

Furthermore, because newer sources of information found on the Internet continue to grow, there is a need to enhance surveillance of new or novel technology and to train and prepare future physicians to recognize the difference between reliable and unreliable health-related sources [17]. The literature shows that health care providers are starting to use personal digital assistants (PDAs) and mobile phone text messaging systems to obtain information $[18,19]$, e-mail patient reminders, and follow up on treatment plans [20] and turning to other, less research-based Web-messaging tools [21], such as blogs, Twitter, Facebook, and other social network Web sites (also known as Web 2.0) for cessation advice. With the expansion of alternative, credible, healthrelated information outlets that are used by physicians, their influence on physician referral patterns and other cessationrelated activities should be explored.

The findings reported in this study may be subject to several limitations. First, the sampling method that was used for the survey was drawn from a self-selected group (Epocrates Honor Panel) and, thus, resulted in a convenience sample. Although the method used quotas and weighting to produce a data set that matched the specialty breakdown of the AMA membership, the findings may not be representative of all US physicians, especially those who were not members of the AMA. Second, 2008 response rates were lower than previous years, which may affect the representativeness of participants. This lower response rate may be a result of the survey being almost twice as long as in previous years, and potential participants were informed of the survey length in the invitational email, which may have dissuaded participation. Third, the questions used to ascertain provider referral to cessation services have not been validated and may not accurately capture providers' actual behavior. Finally, the sample size for some of the racial/ethnic groups was too small to generate a stable estimate and women were underrepresented in the study.

Physicians are essential frontline treatment providers for smoking cessation services, and they obtain medical information from a variety of trusted venues: medical journals, government health agencies, physician colleagues, professional medical associations, and medical Web sites or podcasts. More research is needed about what influences referral patterns to examine why those who depend on professional medical associations for health information are more likely to refer patients to cessation services than those who do not list medical associations as a trusted source of information. Future studies are needed to expand the list of health-related 


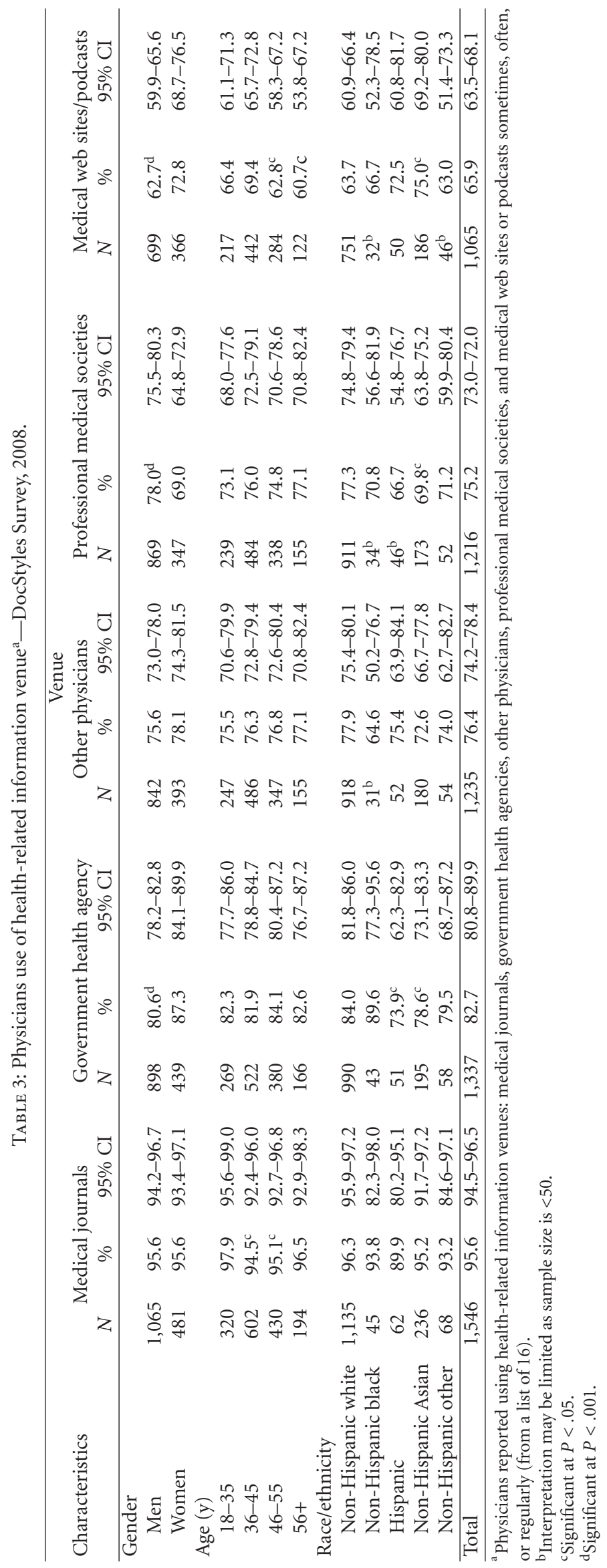


TABLE 4: Logistic regression of physician referral to cessation services ${ }^{\mathrm{a}}$ by most trusted health-related information ${ }^{\mathrm{b}}$.

\begin{tabular}{|c|c|c|c|c|}
\hline Use of most trusted health-related information & Unadjusted $\mathrm{OR}^{\mathrm{c}}$ & $95 \% \mathrm{CI}$ & Adjusted $\mathrm{OR}^{\mathrm{d}}$ & $95 \% \mathrm{CI}$ \\
\hline \multicolumn{5}{|l|}{ Medical journals } \\
\hline No & Reference & & Reference & \\
\hline Yes & 1.15 & $0.71-1.87$ & 1.24 & $(0.76-2.03)$ \\
\hline \multicolumn{5}{|l|}{ Government health agencies } \\
\hline No & Reference & & Reference & \\
\hline Yes & 0.94 & $0.71-1.22$ & 0.96 & $(0.74-1.24)$ \\
\hline \multicolumn{5}{|l|}{ Other physicians } \\
\hline No & Reference & & Reference & \\
\hline Yes & 1.20 & $0.95-1.51$ & 1.23 & $(0.97-1.55)$ \\
\hline \multicolumn{5}{|l|}{ Professional medical societies } \\
\hline No & Reference & & Reference & \\
\hline Yes & 1.29 & $1.02-1.63^{\mathrm{e}}$ & 1.31 & $(1.03-1.66)^{\mathrm{e}}$ \\
\hline \multicolumn{5}{|l|}{ Medical Web sites/podcasts } \\
\hline No & Reference & & Reference & \\
\hline Yes & 1.25 & $1.01-1.54^{\mathrm{e}}$ & 1.22 & $(0.99-1.51)$ \\
\hline $\begin{array}{l}\text { a Cessation services consist of telephone quitline, a smo } \\
\text { b Physicians reported using the } 5 \text { most trusted health-re } \\
\text { c OR refers to odds ratio. Separate logistic regression m } \\
\text { source and referral to cessation services. } \\
\text { d OR is adjusted for sex, age, and race/ethnicity. } \\
\text { e } \text { Significant at } P<.05 \text {. }\end{array}$ & $\begin{array}{l}\text { ssation class, or one- } \\
\text { nformation venues fro } \\
\text { were used to test the }\end{array}$ & $\begin{array}{l}\text { ounseling. } \\
\text { of } 16 . \\
\text { hip between }\end{array}$ & most trusted h & information \\
\hline
\end{tabular}

information venues and include novel or new information technology outlets that physicians use and trust.

Several medical organizations have initiatives to promulgate the 5 A's. Groups that work to disseminate guidelines and best practices in tobacco control should consider working with specialty societies to disseminate their messages. Because the health-related information sources that physicians use can affect their referral patterns, health communicators wishing to reach physicians with quitline cessation information should consider using multiple venues when developing their communication plans.

\section{Disclosure}

J. Kruger has no financial disclosures. A. Trosclair has no financial disclosures. C. Bruce has no financial disclosures. D. Beistle has no financial disclosures.

\section{Disclaimer}

The findings and conclusions in this report are those of the authors and do not necessarily represent the official position of the Centers for Disease Control and Prevention.

\section{Acknowledgments}

Special thanks are due to Dr. Deanne L. Weber, Senior Vice President Strategic Planning and Research, Porter Novelli, and Dr. William Pollard (Division of Communication Services, Centers for Disease Control and Prevention) for providing technical guidance.

\section{References}

[1] CDC, "Vital signs: current cigarette smoking among adults aged $\geq 18$ years-United States, 2005-2010," Morbidity and Mortality Weekly Report (MMWR), vol. 60, no. 35, pp. 12071212, 2011.

[2] Association of American Medical Colleges, "Physician behavior and practice patterns related to smoking cessation: Full report," Washington, DC, USA, pp. 1-146, 2007, http://www .legacyforhealth.org/PDFPublications/Physicians_Study.pdf.

[3] M. C. Fiore, C. R. Jaen, T. B. Baker et al., Treating Tobacco Use and Dependence: 2008 Update, Clinical Practice Guideline, Public Health Service, Rockville, Md, USA, 2008.

[4] Partnership for Prevention, "Healthcare provider reminder systems, provider education and patient education: Working with healthcare delivery systems to improve the delivery of tobacco-use treatment to patients-An action guide. The Community Health Promotion Handbook: Action Guides to Improve Community Health," Washington, DC, USA, 2008, http://www.prevent.org/Initiatives/Action-Guides.aspx.

[5] V. Lemmens, A. Oenema, I. K. Knut et al., "Effectiveness of smoking cessation interventions among adults: a systematic review of reviews," European Journal of Cancer Prevention, vol. 17, no. 6, pp. 535-544, 2008.

[6] R. D. Hurt, J. O. Ebbert, J. T. Hays, and D. D. McFadden, "Treating tobacco dependence in a medical setting," A Cancer Journal for Clinicians, vol. 59, no. 5, pp. 314-326, 2009.

[7] J. Cornuz, W. A. Ghali, D. Di Carlantonio, A. Pecoud, and F. Paccaud, "Physicians attitudes towards prevention: importance of intervention-specific barriers and physicians health habits," Family Practice, vol. 17, no. 6, pp. 535-541, 2000.

[8] AHRQ, "Information to guide physician practice," no. PB97500250, National Technical Information Service (NTIS), Springfield, VA, USA,1996, http://www.ahrq.gov/research/ physprac.htm. 
[9] J. E. Andrews, K. A. Pearce, C. Ireson, and M. M. Love, "Information-seeking behaviors of practitioners in a primary care practice-based research network (PBRN)," Journal of the Medical Library Association, vol. 93, no. 2, pp. 206-212, 2005.

[10] P. Wilson, M. Petticrew, and A. Booth, "After the gold rush? A systematic and critical review of general medical podcasts," Journal of the Royal Society of Medicine, vol. 102, no. 2, pp. 6974, 2009.

[11] J. E. Andrews, K. A. Pearce, C. Sydney, C. Ireson, and M. Love, "Current state of information technology use in a US primary care practice-based research network," Informatics in Primary Care, vol. 12, no. 1, pp. 11-18, 2004.

[12] C. C. Revell and S. A. Schroeder, "Simplicity matters: using system-level changes to encourage clinician intervention in helping tobacco users quit," Nicotine and Tobacco Research, vol. 7, no. 1, pp. S67-S69, 2005.

[13] American Academy of Pediatrics, "Policy statement-tobacco use: a pediatric disease," Pediatrics, vol. 124, pp. 1474-1475, 2009.

[14] American College of Obstetricians and Gynecologists, "Committee opinion, no. 471," Obstetrics \& Gynecology, vol. 116, no. 2, pp. 1241-1244, 2010.

[15] AMA, "Report of the council on medical service," CMS Report 1- I-98, AMA Policy Compendium Subcommittee, Chicago, IL, USA, 1998, www.ama-assn.org/resources/doc/ cms/i98cms1.doc"

[16] American Academy of Family Physicians, "Tobacco use and prevention-Position paper," 2012, http://www.aafp.org/ online/en/home/policy/policies/t/tobaccoprevcess.html.

[17] B. Hughes, I. Joshi, H. Lemonde, and J. Wareham, "Junior physician's use of Web 2.0 for information seeking and medical education: a qualitative study," International Journal of Medical Informatics, vol. 78, no. 10, pp. 645-655, 2009.

[18] K. Terry, "Doctors are getting more tech savvy," Medical Economics, vol. 84, no. 15, pp. 52-56, 2007.

[19] C. Free, R. Whittaker, R. Knight, T. Abramsky, A. Rodgers, and I. G. Roberts, "Txt2stop: a pilot randomised controlled trial of mobile phone-based smoking cessation support," Tobacco Control, vol. 18, no. 2, pp. 88-91, 2009.

[20] M. R. Patt, T. K. Houston, M. W. Jenckes, D. Z. Sands, and D. E. Ford, "Doctors who are using e-mail with their patients: a qualitative exploration," Journal of Medical Internet Research, vol. 5, no. 2, pp. e9-e26, 2003.

[21] E. M. Liederman and C. S. Morefield, "Web messaging: a new tool for patient-physician communication," Journal of the American Medical Informatics Association, vol. 10, no. 3, pp. 260-270, 2003. 


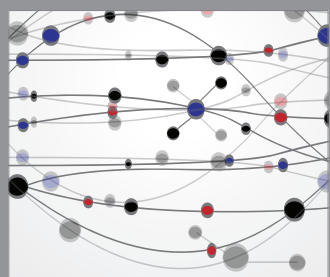

The Scientific World Journal
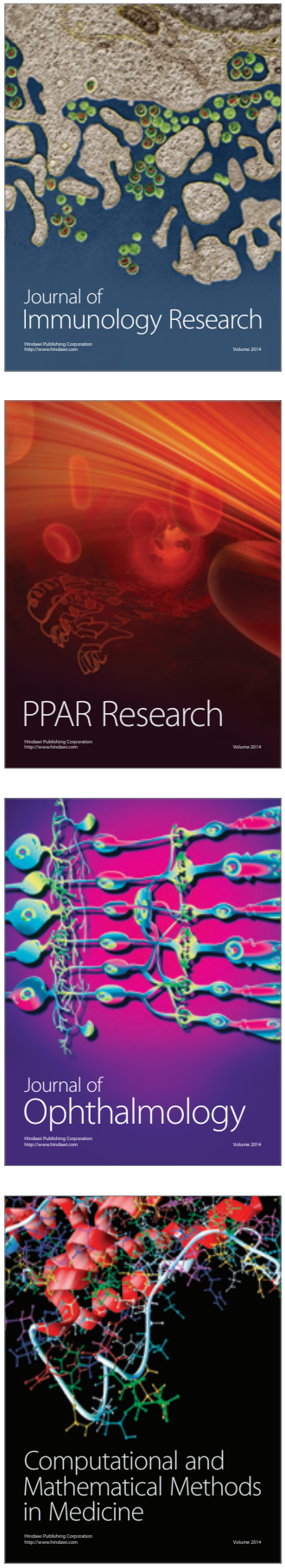

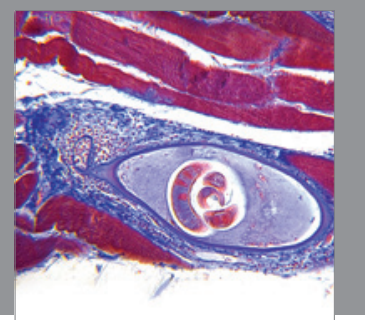

Gastroenterology

Research and Practice
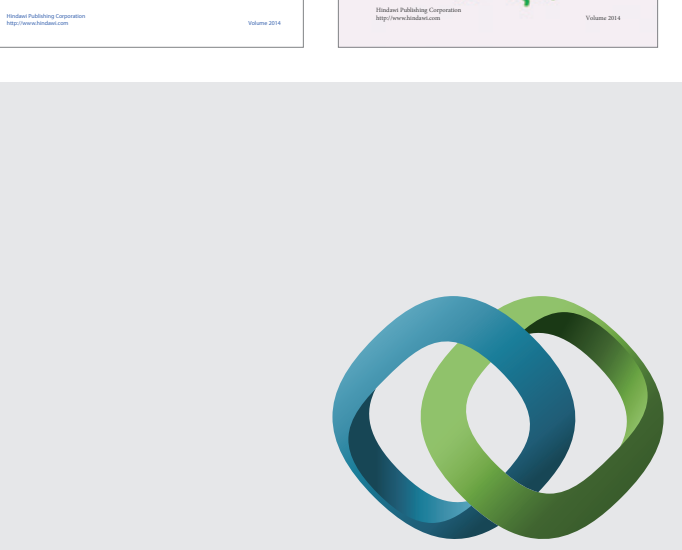

\section{Hindawi}

Submit your manuscripts at

http://www.hindawi.com
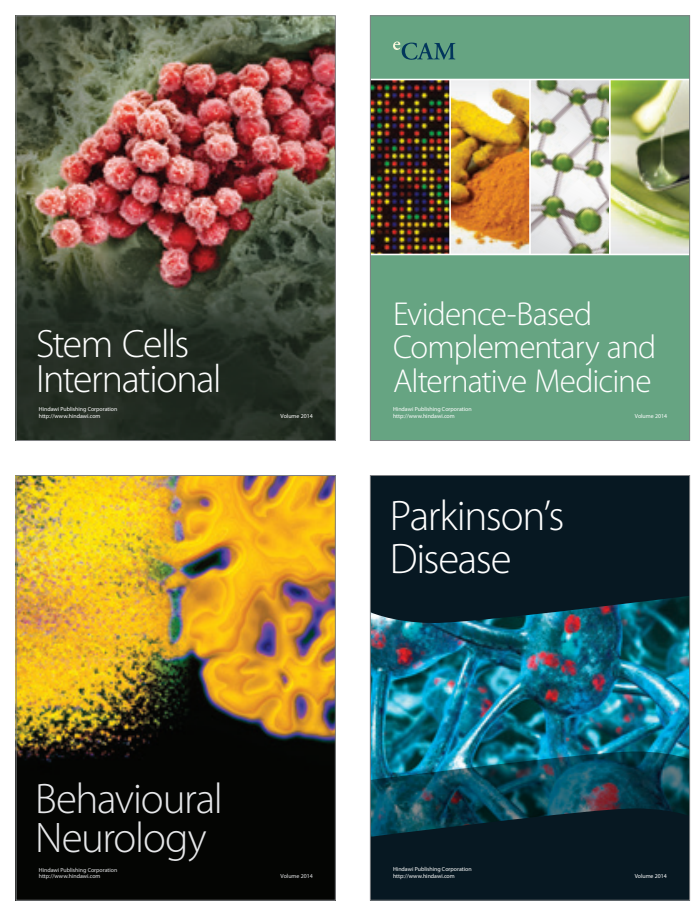

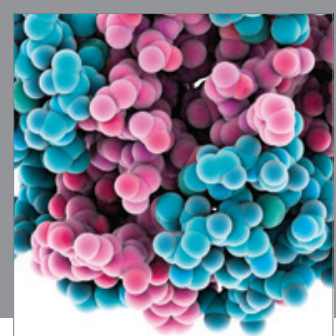

Journal of
Diabetes Research

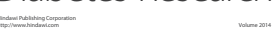

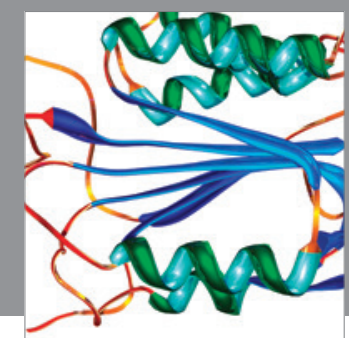

Disease Markers
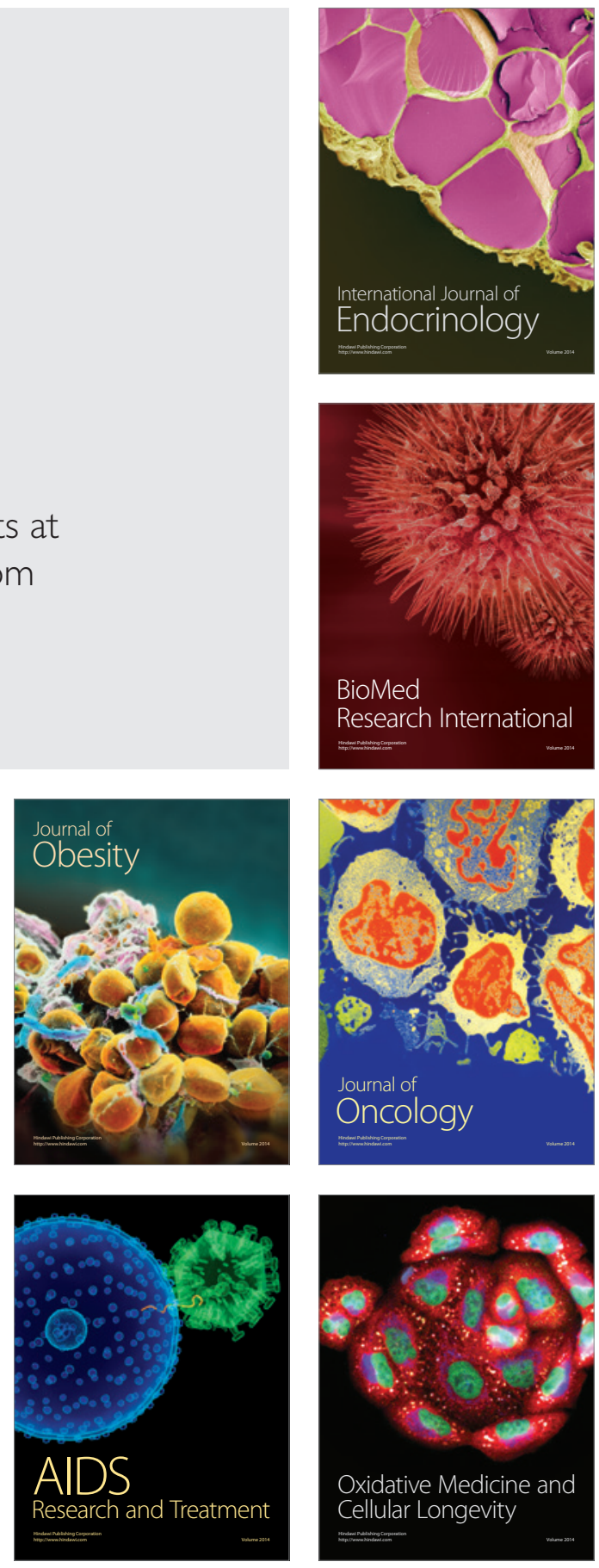